\title{
openheart Safety and effectiveness of non-vitamin $K$ oral anticoagulants versus warfarin in real-world patients with non-valvular atrial fibrillation: a retrospective analysis of contemporary Japanese administrative claims data
}

\author{
Shun Kohsaka, ${ }^{1}$ Jun Katada (D) , ${ }^{2}$ Kumiko Saito, ${ }^{3}$ Aaron Jenkins, ${ }^{4}$ Benjamin $\mathrm{Li}^{5}$ \\ Jack Mardekian, ${ }^{5}$ Yasuo Terayama ${ }^{6}$
}

\begin{abstract}
- Additional material is published online only. To view please visit the journal online (http://dx.doi.org/10.1136/ openhrt-2019-001232).
\end{abstract}

To cite: Kohsaka S, Katada J, Saito K, et al. Safety and effectiveness of non-vitamin $\mathrm{K}$ oral anticoagulants versus warfarin in real-world patients with non-valvular atrial fibrillation: a retrospective analysis of contemporary Japanese administrative claims data. Open Heart 2020;7:e001232. doi:10.1136/ openhrt-2019-001232

Received 17 December 2019 Revised 17 February 2020 Accepted 27 February 2020

Check for updates

C) Author(s) (or their employer(s)) 2020. Re-use permitted under CC BY. Published by BMJ.

For numbered affiliations see end of article.

Correspondence to Dr Jun Katada; jun.katada@ pfizer.com

\section{ABSTRACT}

Objective To assess the safety (ie, risk of bleeding) and effectiveness (ie, risk of stroke/systemic embolism (SE)) separately for four non-vitamin $\mathrm{K}$ oral anticoagulants (NOACs; apixaban, dabigatran, edoxaban and rivaroxaban) versus warfarin in Japanese patients with non-valvular atrial fibrillation (NVAF), including those at high risk of bleeding and treated with reduced doses of NOACs.

Methods We conducted a retrospective analysis of electronic health records and claims data from 372 acute care hospitals in Japan for patients with NVAF newly initiated on NOACs or warfarin. Baseline characteristics were balanced using inverse probability of treatment weighting with stabilised weights (s-IPTW). Bleeding risk and stroke/SE risk were expressed as HRs with 95\% Cls. Two sensitivity analyses were conducted.

Results A total of 73989 patients were eligible for analysis. Notably, $52.8 \%-81.9 \%$ of patients received reduced doses of NOACs. After applying s-IPTW, patient characteristics were well balanced across warfarin/NOAC cohorts. The mean within-cohort age, CHADS, score and $\mathrm{CHA}_{2} \mathrm{DS}_{2}$-VASc score were 76 years, 2.2-2.3 and 3.8, respectively. In all age categories, the majority of the HRs for major bleeding, any bleeding and stroke/SE were equal to or below 1 for all NOACs versus warfarin. Apixaban was the only NOAC associated with a significantly lower risk of any bleeding. There was a trend towards increased risk reduction with NOACs versus warfarin in patients with body weight $\geq 60 \mathrm{~kg}$. In patients with renal disease, the HRs for apixaban versus warfarin were below 1 for major bleeding, any bleeding and stroke/SE, with statistical significance observed for the risk reduction in stroke/SE versus warfarin. In the sensitivity analysis, there were no large differences in HRs between the two observational periods.

Conclusions In patients with NVAF primarily treated with reduced-dose NOACs, the risks of stroke/SE and major bleeding were significantly lower with NOACs versus warfarin.

\section{Key questions}

What is already known about this subject?

- For the prevention of stroke and systemic embolism (SE) in patients with non-valvular atrial fibrillation (NVAF), clinical guidelines recommend treatment with non-vitamin $\mathrm{K}$ oral anticoagulants (NOACs) rather than warfarin. However, the effectiveness and safety of NOACs in Japanese clinical practice remain to be fully elucidated, particularly in patients with high-risk profiles compared with those enrolled in clinical trials.

What does this study add?

- This study found that the majority of patients with NVAF treated in Japanese clinical practice received reduced doses of NOACs-a treatment pattern likely underpinned by bleeding-related concerns. Despite the dose reduction, the risks of stroke/SE, major bleeding and major intracranial haemorrhage were significantly lower for NOACs versus warfarin in Japanese patients with NVAF.

How might this impact on clinical practice?

- These findings provide important real-world evidence describing treatment patterns and clinical outcomes for elderly patients with NVAF treated in Japanese clinical practice. They indicate that NOAC treatment was associated with clinical benefits versus warfarin, even in a population primarily treated with reduced doses.

\section{INTRODUCTION}

Atrial fibrillation (AF) is the most common arrhythmia and is observed in $<1 \%$ of the total population in Japan. ${ }^{1}$ The prevalence of $\mathrm{AF}$ increases with age, rising to approximately $14 \%$ in patients aged $>80$ years. ${ }^{12}$ $\mathrm{AF}$ is a well-established risk factor for stroke, systemic embolism (SE) and death. ${ }^{34}$ Recent 
guidelines recommend treatment with non-vitamin $\mathrm{K}$ oral anticoagulants (NOACs) (ie, apixaban, dabigatran, edoxaban and rivaroxaban) for eligible oral anticoagulant (OAC)-naïve patients with non-valvular atrial fibrillation (NVAF) ${ }^{25}$ Multiple randomised controlled trials (RCTs) have supported the benefits of NOACs versus warfarin in patients with $\mathrm{NVAF}^{6-9}$ with a meta-analysis confirming that NOACs significantly lower the risk of stroke/SE with a risk of major bleeding similar to that associated with warfarin. ${ }^{10}$

While RCTs are the gold standard for demonstrating the effectiveness of interventions, they are not fully representative of an unselected real-world population, thereby limiting the relevance of their findings to clinical practice. Consequently, a number of observational, real-world evidence studies have emerged to provide supportive evidence of the safety and/or effectiveness of NOACs in clinical practice. ${ }^{11-18}$ However, there remain several unmet knowledge gaps in the literature regarding the clinical outcomes of NOAC treatment in patients with NVAF, particularly in patient subgroups at high risk of adverse outcomes. ${ }^{1920}$

All four NOACs (apixaban, dabigatran, edoxaban and rivaroxaban) have been approved in Japan for the prevention of stroke and SE in patients with $\mathrm{NVAF}^{21}$ Importantly, dosing of NOACs in Japan differs slightly from that in other countries given the higher bleeding complication rates reported in East Asian patients; for example, the approved dose of rivaroxaban is $10 / 15 \mathrm{mg}$ daily in Japan. ${ }^{21}$ Given the unique setting surrounding the use of NOACs, and considering they are often initiated at reduced doses, the impact of NOACs on safety (ie, the risk of bleeding) and effectiveness (ie, the risk of stroke or SE) outcomes in Japanese patients with NVAF requires further elucidation.

\section{METHODS}

\section{Study design}

This was a non-interventional, retrospective, observational study conducted from March 2011 (ie, when the first NOAC, dabigatran, was approved in Japan) to July 2018 to evaluate the safety and effectiveness of apixaban, dabigatran, edoxaban and rivaroxaban, each separately, versus warfarin in Japanese patients with NVAF. Written consent from study participants was not necessary in a retrospective study using an existing structured database according to the Japanese Ethical Guidelines. All data were anonymised, and any information that could be used to identify individuals or hospitals was removed.

We used deidentified health claims data from 372 acute care hospitals across Japan available from the Medical Data Vision Co Ltd (MDV; Tokyo, Japan) database. ${ }^{22}$ In brief, the MDV database comprises administrative data for approximately 24 million individuals in the inpatient and outpatient settings. ${ }^{22}$ Each patient is assigned a specific ID to which all inpatient and outpatient data are linked. The distribution of demographic characteristics, including age and sex, of patients registered in the
MDV database is very similar to the national population statistics in Japan. For each prescription recorded in the MDV database, the diagnosis is listed according to 10th Revision of the International Classification of Diseases (ICD-10) codes or local disease codes.

Patients registered in the MDV database between 1 March 2011 and 31 July 2018 were selected based on the following inclusion criteria: diagnosis of $\mathrm{AF}$ at any time during the preindex period and first prescription of any OAC (apixaban, dabigatran, edoxaban, rivaroxaban or warfarin) after a diagnosis of AF; age 18 years or older on the index date (defined as the date of the first prescription of any $\mathrm{OAC}$ ); and no OAC prescription during the year preceding the index date (baseline period). The first OAC prescription recorded in the database was used to identify the patient's index date, treatment cohort and OAC dose. Patients with a diagnosis of valvular AF, postoperative $\mathrm{AF}, \mathrm{AF}$ associated with mechanical valve malfunction, $\mathrm{AF}$ associated with mechanical complication of heart valve prosthesis or rheumatic AF during the baseline period were excluded. Additionally, patients with a diagnosis of hyperthyroidism or thyrotoxicosis, those who underwent procedures involving prosthetic heart valves performed during the baseline period and those with haemodialysis or pregnancy during the baseline period were also excluded.

Patients were followed from the index date until any of the following events, whichever occurred first: discontinuation of the index OAC, defined as a continuous gap of 45 days or more between the expected refill date and the actual refill date; switch to another $\mathrm{OAC}$-if the index $\mathrm{OAC}$ was discontinued and another OAC was started within 45 days of the prescription refill date of the index OAC; lack of further records in the database-if no further relevant records were added (eg, no further refills or visits), the last date of the patient's record in the database was used; occurrence of stroke, SE or haemorrhagic adverse events; or an elapse of 2 years from the index date.

\section{Endpoints}

Individual NOACs and warfarin were compared with respect to the incidence of stroke/SE and bleeding in cohorts after inverse probability of treatment weighting with stabilised weights (s-IPTW) was applied. The safety endpoints were major bleeding and any bleeding, defined as bleeding requiring hospitalisation (major bleeding) and any bleeding event recorded after the index date regardless of severity or need for hospitalisation (any bleeding). Bleeding sites were not considered in the primary analysis. The effectiveness primary endpoint was a composite of stroke and SE requiring hospitalisation. Stroke was defined as ischaemic or haemorrhagic stroke. Haemorrhagic stroke was included both as a safety endpoint and as an effectiveness endpoint. For the secondary analyses, major gastrointestinal (GI) bleeding, any GI bleeding, major intracranial haemorrhage (ICH) and any ICH were the safety-related secondary endpoints. Ischaemic stroke, haemorrhagic stroke and SE were the 
effectiveness-related secondary endpoints. The primary safety and effectiveness endpoints were also assessed in the following prespecified subgroups: age ( $\geq 75$ years $/<75$ years or $\geq 80$ years $/<80$ years $)$, body weight $(\geq 60 \mathrm{~kg} /<60$ $\mathrm{kg}$ ), renal disease (yes/no), concomitant use of antiplatelet drugs (yes/no) and NOAC dose (standard/ reduced). Similar to the primary analyses, s-IPTW was applied to balance patient characteristics among these subgroups. The ICD-10 codes and disease codes used in the study are listed in online supplementary tables 1 and 2.

\section{Statistics}

All analyses were conducted with SAS V.9.4. A propensity score was calculated based on multinomial logistic regression in order to account for confounding effects and to ensure that patient characteristics were balanced between the NOAC and warfarin cohorts. An IPTW method using the calculated propensity score was applied, and to avoid sample size inflation and ensure appropriate estimation of variances, s-IPTW was used. ${ }^{23}{ }^{24}$ Weight truncation was not conducted. The following clinical and demographic characteristics, collected during the baseline period or at the index date, were included as covariates to calculate the propensity score: sex and age, comorbidities (ie, heart failure, coronary heart disease, peripheral vascular disorder, myocardial infarction, stroke, transient ischaemic attack, SE, renal dysfunction, hepatic dysfunction, bleeding history, hypertension and diabetes mellitus), concomitant medications (ie, antiplatelet drugs, nonsteroidal anti-inflammatory drugs, gastric secretion inhibitors, statins, heparins and antihypertensive drugs) and presence of cardioversion and ablation procedures. $\mathrm{CHADS}_{2}$ and $\mathrm{CHA}_{2} \mathrm{DS}_{2}$-VASc scores were calculated using these clinical and demographic characteristics. ${ }^{25}{ }^{26}$ The calculated s-IPTW was simultaneously applied to the five crude OAC cohorts to obtain four paired NOAC/warfarin cohorts, wherein demographic and clinical characteristics of each OAC cohort were balanced. The covariate balance between the NOAC/ warfarin cohorts after s-IPTW was assessed with respect to standardised differences using a threshold of 0.1 ; previous studies have suggested that a standardised difference of $>0.1$ may indicate the presence of a meaningful imbalance of covariates between paired treatments. ${ }^{27-29}$ The 2-year cumulative incidence rates of major bleeding, any bleeding and stroke/SE in the cohorts after s-IPTW were plotted with Kaplan-Meier curves. HRs with 95\% CIs were calculated using a Cox proportional hazards regression model that incorporated only the index OACs as independent variables.

\section{Sensitivity analyses}

Two sensitivity analyses were conducted. First, a sensitivity analysis was performed by restricting the follow-up period to 1 year, and differences in the results versus the 2-year follow-up period were compared. Second, a conventional 1:1 propensity score matching method was used to assess the robustness of the method used for addressing the covariate imbalance between cohorts. As in the main analysis, a threshold of 0.1 was used for confirming covariate balance between the two groups, and HRs with 95\% CIs were calculated using a Cox proportional hazards model.

\section{RESULTS}

\section{Baseline characteristics in the crude cohorts before s-IPTW}

Overall, 73989 patients were eligible for the analysis after applying the selection criteria (figure 1). Patients were divided into five cohorts: 15902 patients initiated warfarin; 22336 patients initiated apixaban $2.5 \mathrm{mg}$ or $5 \mathrm{mg}$ twice daily; 6925 patients initiated dabigatran $110 \mathrm{mg}$ or $150 \mathrm{mg}$ twice daily; 12262 patients initiated edoxaban $30 \mathrm{mg}$ or $60 \mathrm{mg}$ once daily; and 16564 patients initiated rivaroxaban $10 \mathrm{mg}$ or $15 \mathrm{mg}$ once daily (figure 1). Baseline characteristics in the crude cohorts before s-IPTW are reported in online supplementary table S3. The mean (SD) duration of treatment ranged from 265 (263.8) to 868 (725) days. Apixaban was the most frequently prescribed NOAC, and $47.2 \%-76.2 \%$ of patients were initiated on reduced doses of NOACs. The warfarin cohort contained the oldest patients, with the highest mean $\mathrm{CHADS}_{2}$ and $\mathrm{CHA}_{2} \mathrm{DS}_{2}$-VASc scores and the most comorbidities, and patients in the apixaban cohort tended to be older with higher mean risk scores (online supplementary table S3).

\section{Incidence of bleeding and stroke/SE in the crude cohorts before s-IPTW}

Between-cohort differences in event rates (per 100 personyears) reflected differences in baseline patient risk characteristics (online supplementary table S3). The event rates of major bleeding, any bleeding and stroke/SE were highest in the warfarin cohort; the event rates of major bleeding and any bleeding were lowest in the dabigatran cohort, and the event rate of stroke/SE was lowest in the rivaroxaban cohort (online supplementary table S4).

\section{Patient characteristics in the cohorts after s-IPTW}

The standardised differences between the s-IPTWbalanced cohorts in patient characteristics used for calculating the propensity score were $<0.1$, suggesting that patient characteristics were well balanced between the cohorts (table 1). For patients treated with dabigatran, the age, $\mathrm{CHADS}_{2}$ and $\mathrm{CHA}_{2} \mathrm{DS}_{2}$-VASc scores and proportion of patients with comorbidities were slightly higher than those in the crude cohort. The proportion of patients treated with reduced doses of NOACs remained high $(52.8 \%-81.9 \%$; table 1$)$.

\section{Bleeding and stroke/SE risk in the cohorts after s-IPTW}

Unweighted Kaplan-Meier cumulative incidence plots of any bleeding, major bleeding and stroke/SE events are presented in figure 2. Compared with warfarin, all NOACs were associated with a significantly lower risk of stroke/ SE and major bleeding (figure 3). Apixaban was associated with a significantly lower risk of any bleeding, and 


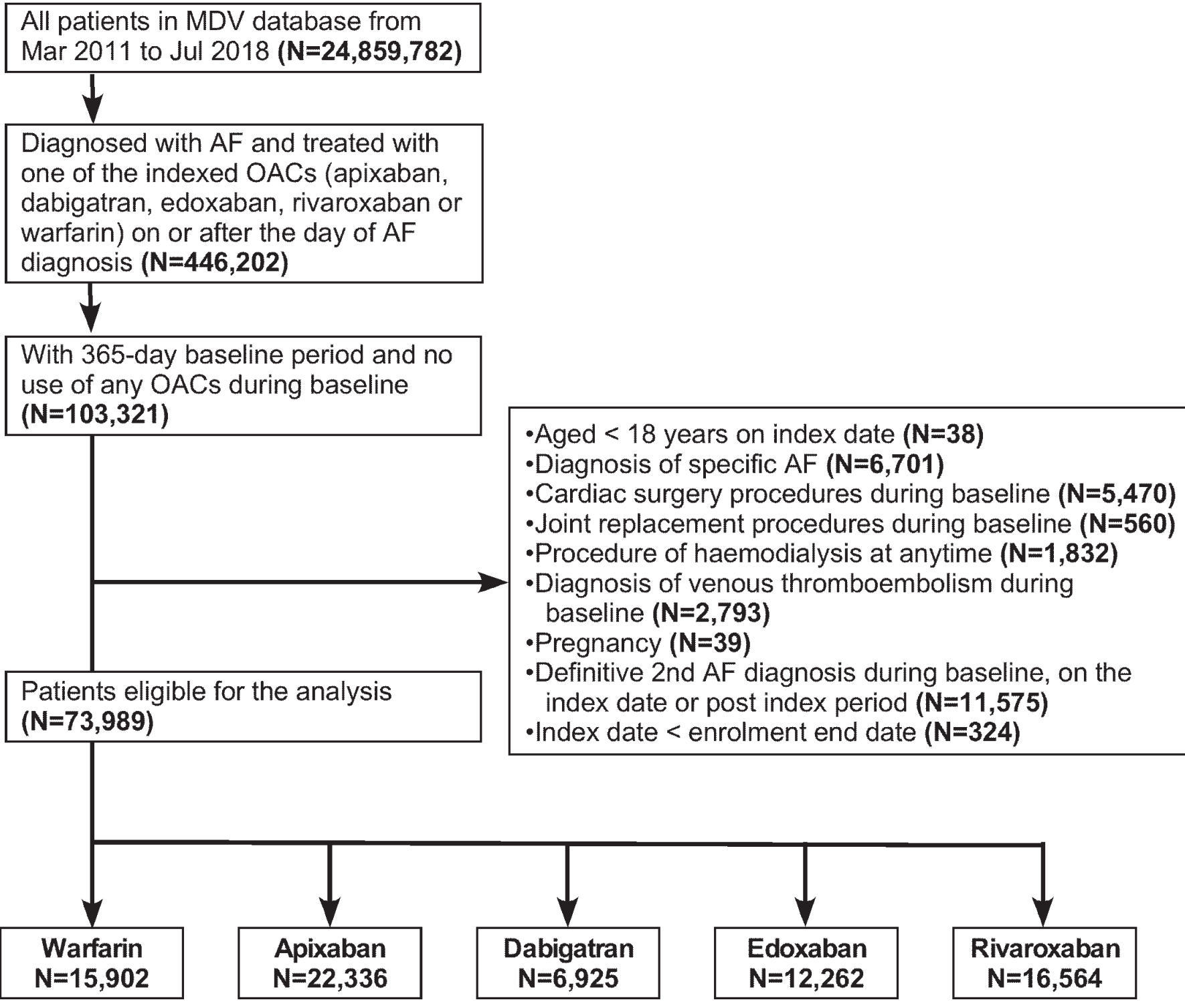

Figure 1 Flow chart of patient allocation to each OAC cohort. AF, atrial fibrillation; MDV, Medical Data Vision Co Ltd; OAC, oral anticoagulant.

dabigatran and rivaroxaban had HRs below 1; however, statistical significance was not achieved (figure 3).

\section{Secondary safety and effectiveness endpoints}

A significantly lower risk of major ICH was observed for all NOACs versus warfarin, and dabigatran and rivaroxaban were associated with a significantly lower risk of any ICH (table 2). Apixaban was associated with a significantly lower risk of major GI bleeding, and apixaban and rivaroxaban were associated with a significantly lower risk of any GI bleeding (table 2). Compared with warfarin, all of apixaban, edoxaban and rivaroxaban were associated with a significantly lower risk of ischaemic stroke, while dabigatran and rivaroxaban were associated with a significantly lower risk of haemorrhagic stroke. All NOACs had HRs below 1 for SE; however, statistical significance was not achieved (table 2).

\section{Subgroup analyses in patients with high-risk profiles}

Online supplementary table S5 reports the results of the subgroup analysis in high-risk patients. Across age categories, the majority of the HRs for major bleeding, any bleeding and stroke/SE were equal to or below 1 for all NOACs versus warfarin, although not all were statistically significant. In the very elderly age group ( $\geq 80$ years), apixaban, edoxaban and rivaroxaban were associated with a significantly lower risk of major bleeding and stroke/SE (online supplementary table S5).

In patients with body weight $<60 \mathrm{~kg}$, apixaban was associated with a significantly lower risk of stroke/SE, and there was a trend towards risk reduction for major bleeding and any bleeding with NOACs versus warfarin in patients with body weight $\geq 60 \mathrm{~kg}$. In patients with renal disease, the HRs for apixaban alone (vs warfarin) were below 1 for major bleeding, any bleeding and stroke/SE, with statistical significance observed for the risk reduction in stroke/SE versus warfarin (online supplementary table S5). 


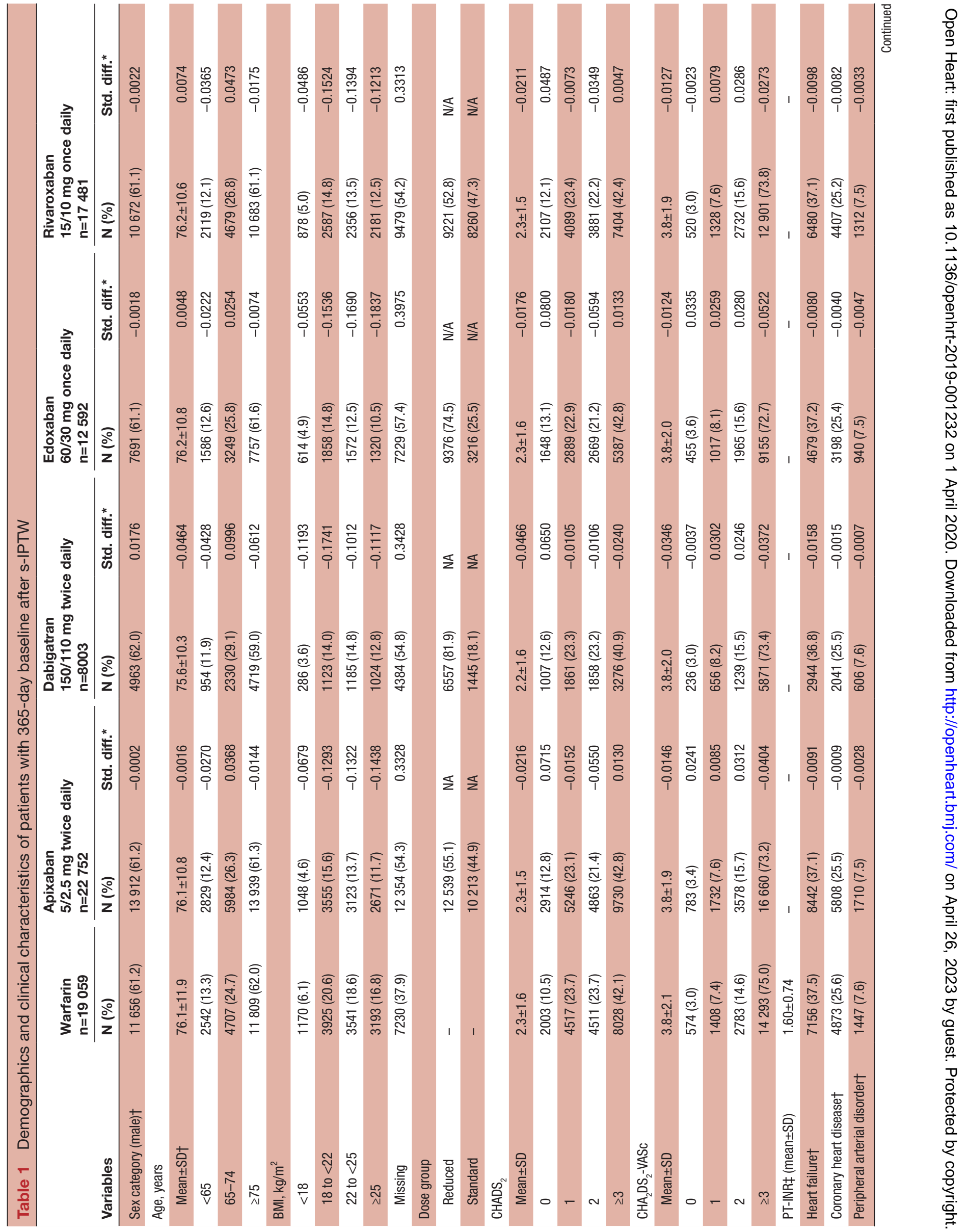




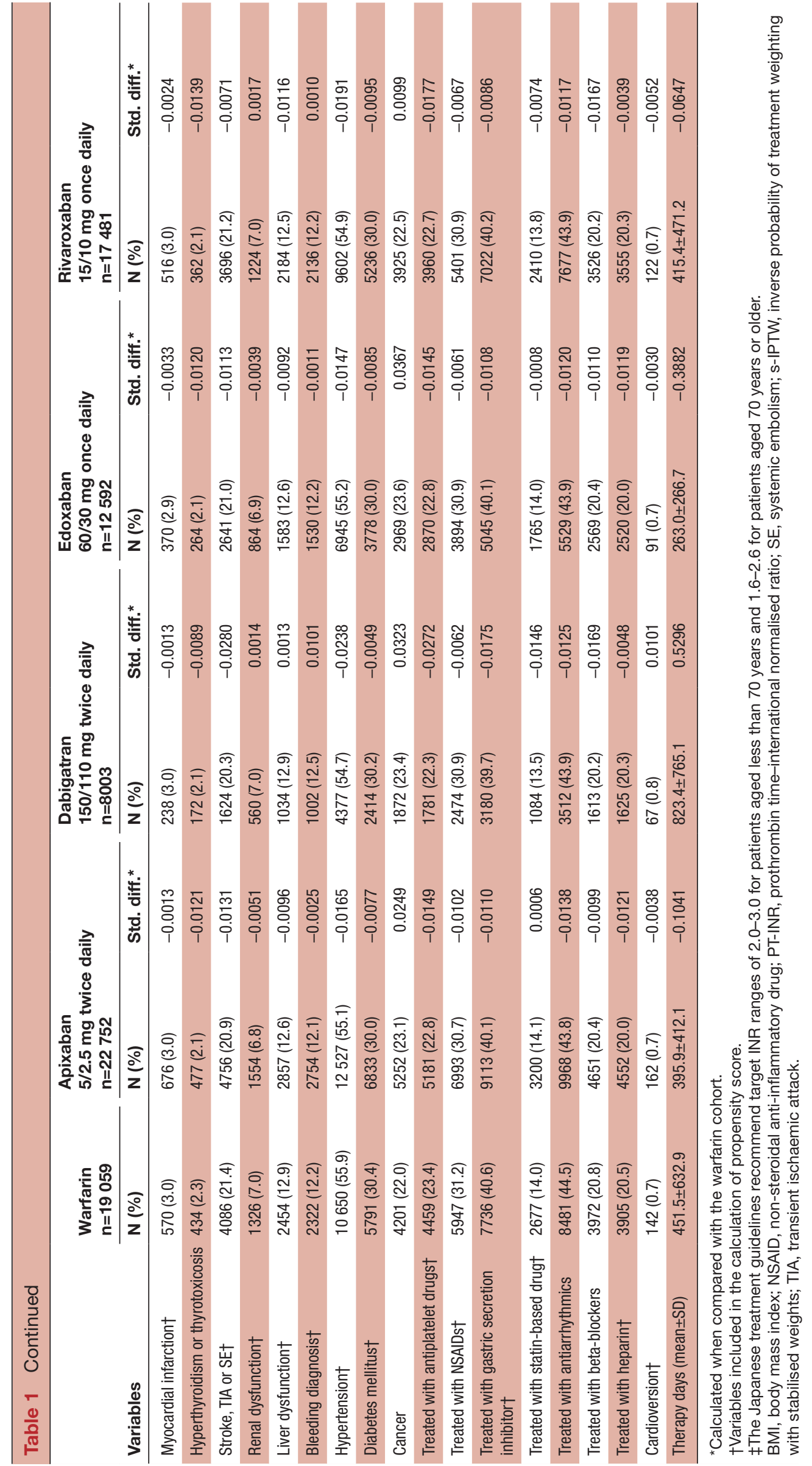



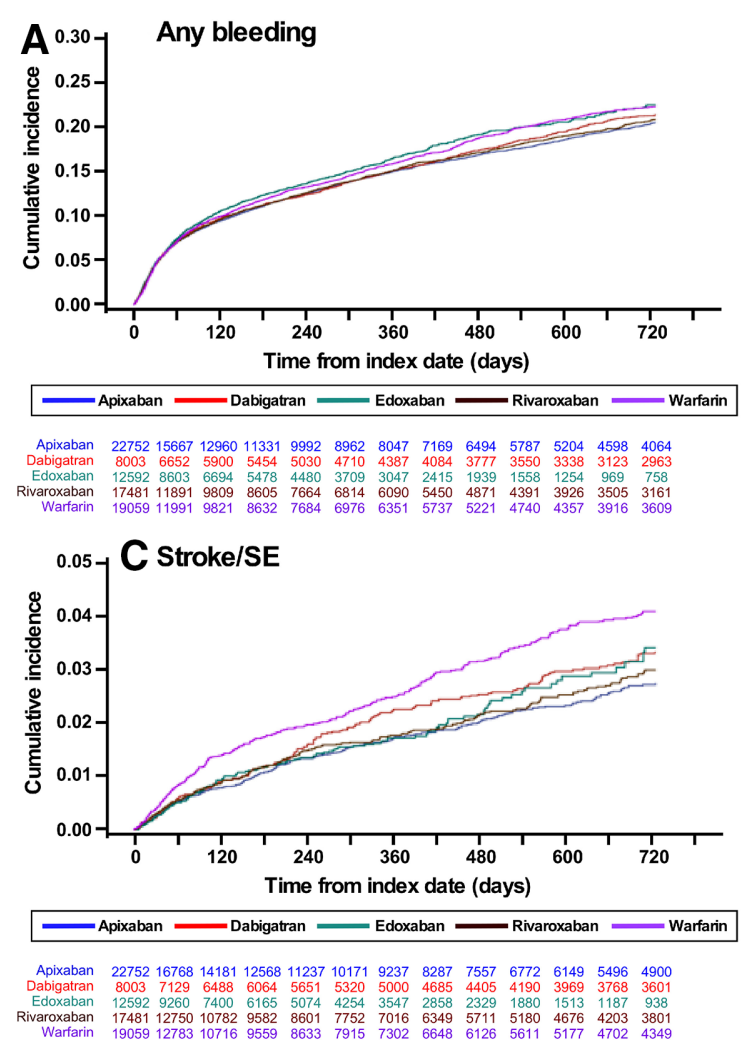

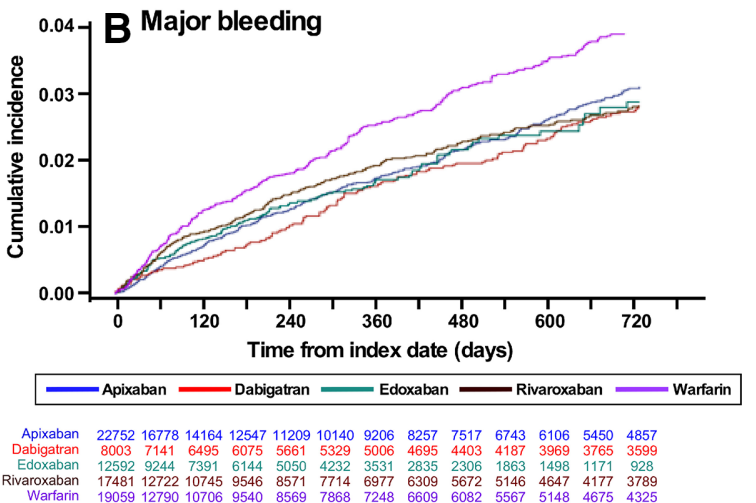

Figure 2 Kaplan-Meier curves for incidence of (A) any bleeding, (B) major bleeding and (C) stroke/SE. SE, systemic embolism.

When stratified by initial dose (ie, standard vs reduced), the risk of any bleeding was significantly higher with the standard dose of edoxaban, and the

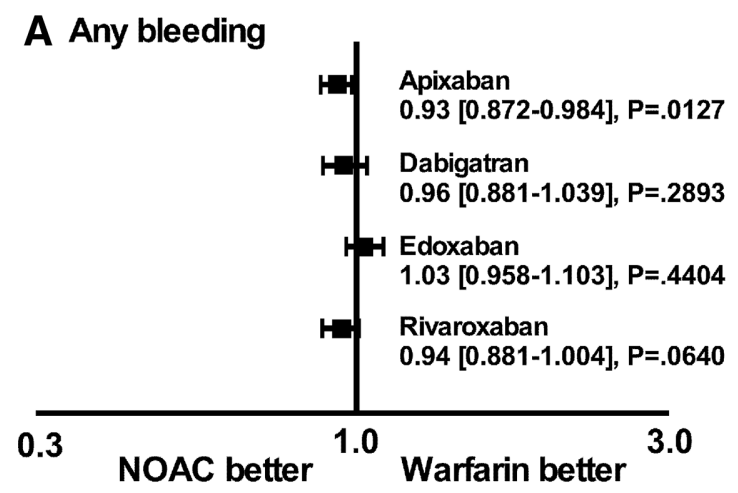

\section{Stroke/SE}

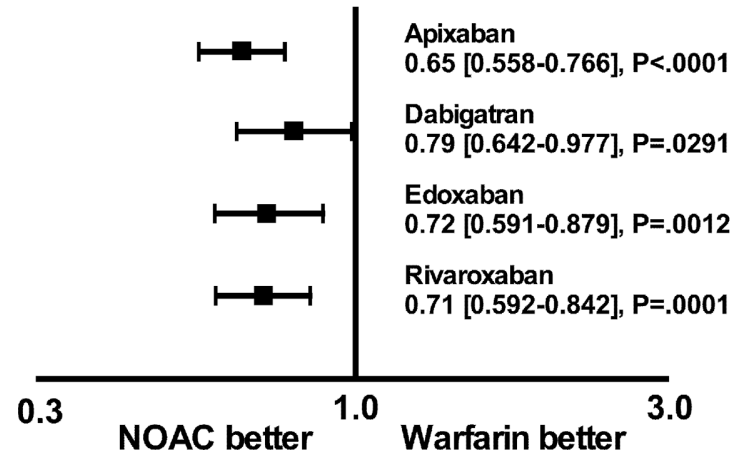

risk of stroke/SE was significantly lower with a reduced dose of apixaban versus warfarin (online supplementary table S5).

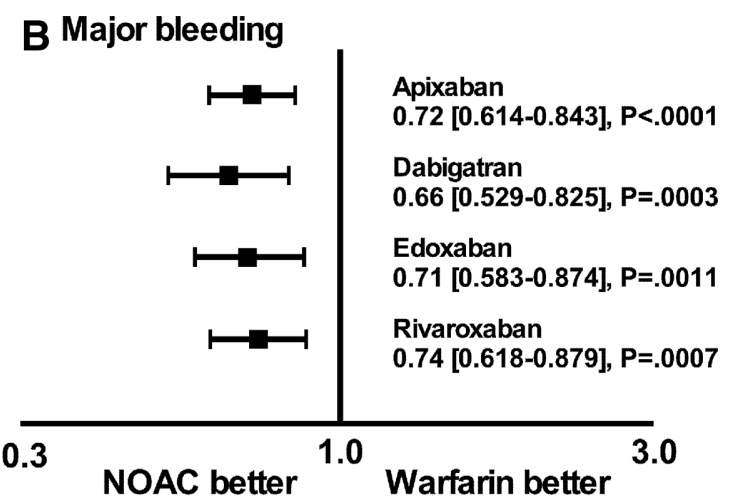

Figure 3 Forest plot depicting the risk of events for NOACs versus warfarin. HRs and 95\% Cls are given for each NOAC. NOAC, non-vitamin K oral anticoagulant; SE, systemic embolism. 


\begin{tabular}{|c|c|c|c|c|c|}
\hline & \multirow{2}{*}{$\begin{array}{l}\mathrm{N} \text { (NOAC/ } \\
\text { warfarin) }\end{array}$} & $\begin{array}{l}\text { Apixaban } \\
5 / 2.5 \text { mg twice daily } \\
\text { over warfarin }\end{array}$ & $\begin{array}{l}\text { Dabigatran } 150 / 110 \\
\text { mg twice daily over } \\
\text { warfarin }\end{array}$ & $\begin{array}{l}\text { Edoxaban } \\
60 / 30 \text { mg once daily } \\
\text { over warfarin }\end{array}$ & $\begin{array}{l}\text { Rivaroxaban } 15 / 10 \\
\text { mg once daily over } \\
\text { warfarin }\end{array}$ \\
\hline & & $22752 / 19059$ & 8 003/19 059 & $12592 / 19059$ & $17481 / 19059$ \\
\hline \multirow[t]{3}{*}{ Ischaemic stroke } & $\mathrm{HR}$ & 0.63 & 0.90 & 0.74 & 0.74 \\
\hline & $95 \% \mathrm{Cl}$ & (0.524 to 0.759 ) & (0.716 to 1.140$)$ & (0.586 to 0.925$)$ & (0.607 to 0.909 ) \\
\hline & $P$ value & $<0.0001$ & 0.3906 & 0.0087 & 0.0039 \\
\hline \multirow[t]{3}{*}{ Haemorrhagic stroke } & $H R$ & 0.75 & 0.41 & 0.73 & 0.63 \\
\hline & $95 \% \mathrm{Cl}$ & (0.545 to 1.029$)$ & (0.244 to 0.703 ) & (0.479 to 1.102$)$ & (0.432 to 0.922$)$ \\
\hline & $P$ value & 0.0743 & 0.0011 & 0.1332 & 0.0175 \\
\hline \multirow[t]{3}{*}{ Systemic embolism } & $H R$ & 0.48 & 0.97 & 0.46 & 0.50 \\
\hline & $95 \% \mathrm{Cl}$ & (0.198 to 1.165$)$ & (0.316 to 2.959 ) & (0.145 to 1.487$)$ & (0.183 to 1.349$)$ \\
\hline & $P$ value & 0.1050 & 0.9519 & 0.1966 & 0.1701 \\
\hline \multirow[t]{3}{*}{ Major Gl bleeding } & $H R$ & 0.76 & 0.92 & 0.83 & 0.92 \\
\hline & $95 \% \mathrm{Cl}$ & (0.579 to 0.987$)$ & (0.655 to 1.286$)$ & (0.602 to 1.158$)$ & (0.693 to 1.213 ) \\
\hline & $P$ value & 0.0394 & 0.6175 & 0.2798 & 0.5425 \\
\hline \multirow[t]{3}{*}{ Any Gl bleeding } & $H R$ & 0.87 & 1.04 & 0.99 & 0.87 \\
\hline & $95 \% \mathrm{Cl}$ & (0.779 to 0.970$)$ & (0.901 to 1.203 ) & (0.871 to 1.125$)$ & (0.768 to 0.976$)$ \\
\hline & $P$ value & 0.0121 & 0.5870 & 0.8812 & 0.0186 \\
\hline \multirow{3}{*}{$\begin{array}{l}\text { Major intracranial } \\
\text { haemorrhage }\end{array}$} & $\mathrm{HR}$ & 0.58 & 0.42 & 0.60 & 0.52 \\
\hline & $95 \% \mathrm{Cl}$ & (0.452 to 0.757$)$ & (0.283 to 0.637$)$ & (0.427 to 0.836$)$ & (0.379 to 0.702$)$ \\
\hline & $P$ value & $<0.0001$ & $<0.0001$ & 0.0026 & $<0.0001$ \\
\hline \multirow{3}{*}{$\begin{array}{l}\text { Any intracranial } \\
\text { haemorrhage }\end{array}$} & $H R$ & 0.89 & 0.79 & 0.92 & 0.81 \\
\hline & $95 \% \mathrm{Cl}$ & (0.781 to 1.010$)$ & (0.658 to 0.946 ) & (0.789 to 1.076 ) & (0.701 to 0.936 ) \\
\hline & $P$ value & 0.0715 & 0.0104 & 0.3014 & 0.0044 \\
\hline
\end{tabular}

GI, gastrointestinal; NOAC, non-vitamin K oral anticoagulant.

\section{Sensitivity analysis}

There were no large differences in HRs between the two different observational periods ( 1 year and 2 years), although statistical significance was not always obtained for the HRs in the 1-year observation period owing to the small number of events (online supplementary table S6). Results of the second sensitivity analysis were also largely consistent with the main results (online supplementary table S7).

\section{DISCUSSION}

In this large, real-world, observational study, we evaluated the effectiveness and safety of four NOACs currently approved for stroke/SE prevention versus warfarin in Japanese patients with NVAF. The primary results indicated that all NOACs were associated with a significantly lower risk of major bleeding and stroke/SE compared with warfarin. Notable results from the secondary analyses were a significantly lower risk of major ICH for all NOACs, and reductions in the risk of any and major GI bleeding with apixaban, versus warfarin. Broadly, these real-world results provide supportive evidence for the existing RCTs that have demonstrated the clinical benefits of NOACs versus warfarin in patients with NVAF. ${ }^{6-10}$ Moreover, the current study builds on the emerging, realworld evidence base for the effectiveness and safety of NOACs in Japanese clinical practice. ${ }^{15-18} 30$
Reduced dosing of NOACs is a pertinent clinical concern as it may impact the safety and/or effectiveness of treatment. ${ }^{1831} 32$ In the current study, risks for bleeding and stroke/SE were generally consistent between the standard-dose and reduced-dose NOAC subgroups, and we observed a significantly lower risk of stroke/SE with reduced-dose apixaban versus warfarin. Thus, the current results differ from recent real-world study results, in which reduced-dose NOAC treatment was associated with increased rates of thromboembolic and major haemorrhagic events, along with stroke/SE and myocardial infarction, in Japanese patients with NVAF. ${ }^{18} 32$ Of note, the proportions of patients initiated on reduced doses of NOACs were higher than those reported in studies conducted in the USA, ${ }^{33-35}$ Korea and Taiwan ${ }^{36-39}$ and in real-world studies in Japan. ${ }^{17} 1840$ It is likely that the high rates of dose reduction observed in the current study were primarily attributable to the risk characteristics of the patient sample. For instance, in a recent cross-sectional analysis of a multicentre outpatient registry in Japan, the independent predictors of NOAC underdosing in newly diagnosed patients with NVAF were older age, concomitant antiplatelet therapy, impaired renal function and prior heart failure or left ventricular dysfunction. ${ }^{40}$

Appropriate use of NOACs in patients with NVAF and comorbid renal disease remains the subject of ongoing 
investigation, ${ }^{41-45}$ and worsening of renal function in patients with $\mathrm{AF}$ is independently associated with ischaemic stroke and haemorrhage. ${ }^{43}$ NOAC-specific differences in renal excretion rates have been observed, ${ }^{44}$ with dabigatran and edoxaban having the greatest dependence on renal elimination compared with apixaban and rivaroxaban. ${ }^{45} \mathrm{~A}$ recent meta-analysis of RCTs and observational studies in patients with renal disease reported that NOACs significantly lowered the risk of ICH, stroke/ SE and major bleeding versus warfarin. ${ }^{42}$ In the current study, apixaban was the only NOAC with a significantly lower risk of stroke/SE, whereas the risk of stroke/SE was significantly higher for rivaroxaban versus warfarin in patients with NVAF and comorbid renal disease. However, owing to the relatively small number of patients with renal disease, along with the observational design of the study, firm conclusions regarding the safety and effectiveness of NOACs in Japanese patients with NVAF and comorbid renal disease should not be made on the basis of these results.

Strengths of the current study's design and results include the MDV database being representative of the Japanese population, the high mean age of the patients (ie, most were very elderly), the large sample size and the inclusion of all four approved NOACs in the analyses. Additionally, a majority of the patients were treated with reduced doses of NOACs, which allowed for the evaluation of effectiveness and safety in patients on reduced doses; however, this also places limitations on the generalisability of the results to patients with NVAF primarily treated with standard doses of NOACs. Furthermore, the study provides much-needed data on the effectiveness and safety of NOACs in Japanese patients with NVAF, as many studies have been conducted in Western populations. However, the study has several limitations. First, data were obtained from a claims database containing information provided by hospitals applying the flat-fee payment system, which are mostly large hospitals responsible for acute care. Therefore, a significant proportion of the patients included were likely in poorer health than the average population requiring hospitalisation, possibly having more comorbidities and a higher risk of stroke/SE and bleeding. Second, the claims data did not include vital signs or laboratory measurements (eg, blood pressure, international normalised ratio values, renal function parameters), which precluded calculation of a HAS-BLED score. ${ }^{46}$ Therefore, we were unable to consider these variables in the calculation of the propensity score, and consequently, there is no guarantee that these characteristics were fully balanced after s-IPTW. Thus, the influence of unexamined confounding factors cannot be fully excluded. Third, we could not provide an estimate of follow-up loss as we had no subsequent data on patients who had visited a different hospital or clinic after being registered with one of the hospitals contributing to the MDV database. This could have led to an underestimation of the incidence of stroke/SE or major bleeding events. Fourth, results of some subgroup analyses are not conclusive owing to the smaller number of patients and lower statistical power. Fifth, primary endpoints were defined as resulting in hospitalisation, which differs from the adjudicated endpoints typically used in RCTs, and we did not include a mortality endpoint. Finally, while the majority of patients received reduced doses of NOACs, we were unable to determine whether this level of dosing was appropriate or if off-label underdosing of NOACs had any impact on the clinical outcomes owing to the unavailability of clinical information in the MDV database.

In conclusion, a large proportion of patients with NVAF initiated treatment with reduced-dose NOACs in contemporary Japanese practice. Despite this, the risks of stroke/SE, along with major bleeding, were significantly lower for NOACs versus warfarin. The results were largely consistent across the patient subgroups with higher risk profiles, such as those with older age, lower body weight and renal disease.

\section{Author affiliations}

${ }^{1}$ Department of Cardiology, Keio University School of Medicine, Tokyo, Japan ${ }^{2}$ Internal Medicine Medical Affairs, Pfizer Japan Inc, Tokyo, Japan

${ }^{3}$ Cardiovascular Medical Department, Bristol-Myers Squibb K.K, Tokyo, Japan ${ }^{4}$ Department of Patient \& Health Impact, Pfizer Inc, New York, New York, USA ${ }^{5}$ Global Biometrics \& Data Management, Pfizer Inc, New York, New York, USA ${ }^{6}$ Neurological Institute, Shonan Keiiku Hospital, Kanagawa, Japan

Acknowledgements The authors wish to thank Medical Data Vision Co Ltd for advice on dataset preparation. Medical writing support, funded by Pfizer, was provided by Liam Gillies, PhD, CMPP, of Cactus Communications.

Contributors SK and YT were involved in designing the study, defining each disease and comorbid condition according to 10th Revision of the International Classification of Diseases codes, interpreting the obtained results and critically reviewing the drafted manuscript. KS was involved in designing the study, managing the project and interpreting the obtained results. JK was involved in designing the study, managing the project, interpreting the obtained results and drafting the manuscript. AJ contributed to planning the study protocol and statistical analysis, managing the project throughout the study, interpreting the results and reviewing the manuscript critically. JM and BL, who are statisticians, carried out the statistical analysis and contributed to the interpretation of results. All authors provided final approval for this version to be published and agreed to be accountable for all aspects of the work.

Funding This study was funded and conducted by Bristol-Myers Squibb Co and Pfizer Inc. Both companies had significant roles in the study design, data collection/ analysis, manuscript preparation and decision to publish.

Competing interests KS is a full-time employee of Bristol-Myers Squibb Co. $\mathrm{JK}, \mathrm{AJ}$ and BL are full-time employees of Pfizer Inc. JM was a full-time employee of Pfizer Inc until January 2020 and currently serves as a Teaching Professor at Rutgers University, New Jersey, USA. SK reports investigator-initiated grant funding from Bayer and Daiichi Sankyo, and personal fees from AstraZeneca, Bayer, BristolMyers Squibb Co, Daiichi Sankyo, Pfizer Inc, Teikoku Seiyaku and Boehringer Ingelheim, outside the submitted work. YT has served as a consultant for BristolMyers Squibb Co and Pfizer Inc.

Patient consent for publication Not required.

Ethics approval The study was conducted in accordance with the Japanese Ethical Guidelines for Medical and Health Research Involving Human Subjects and was approved by the independent institutional review board (IRB) of Takahashi Clinic as a central IRB (approval date: July 17, 2018).

Provenance and peer review Not commissioned; externally peer reviewed.

Data availability statement Data may be obtained from a third party and are not publicly available. Raw data used for this analysis are not available owing to the 
terms of the contract between Pfizer Japan Inc and Medical Data Vision Co Ltd. If access to the raw data is necessary, please make direct contact with Medical Data Vision Co Ltd. The authors declare that all other supporting data, including the definitions of the diseases, are available within the article and its online supplementary files.

Open access This is an open access article distributed in accordance with the Creative Commons Attribution 4.0 Unported (CC BY 4.0) license, which permits others to copy, redistribute, remix, transform and build upon this work for any purpose, provided the original work is properly cited, a link to the licence is given, and indication of whether changes were made. See: https://creativecommons.org/ licenses/by/4.0/.

ORCID iD

Jun Katada http://orcid.org/0000-0003-3588-3686

\section{REFERENCES}

1 Inoue $\mathrm{H}$, Fujiki $\mathrm{A}$, Origasa $\mathrm{H}$, et al. Prevalence of atrial fibrillation in the general population of Japan: an analysis based on periodic health examination. Int J Cardiol 2009;137:102-7.

2 JCS Joint Working Group. Guidelines for pharmacotherapy of atrial fibrillation (JCS 2013). Circ J 2014;78:1997-2021.

3 Wolf PA, Abbott RD, Kannel WB. Atrial fibrillation as an independent risk factor for stroke: the Framingham study. Stroke 1991;22:983-8.

4 Fuster V, Rydén LE, Cannom DS, et al. ACC/AHA/ESC 2006 guidelines for the management of patients with atrial FibrillationExecutive summary. Circulation 2006;114:700-52.

5 Steffel J, Verhamme P, Potpara TS, et al. The 2018 European heart rhythm association practical guide on the use of non-vitamin $\mathrm{K}$ antagonist oral anticoagulants in patients with atrial fibrillation. Eur Heart J 2018;39:1330-93.

6 Connolly SJ, Ezekowitz MD, Yusuf S, et al. Dabigatran versus warfarin in patients with atrial fibrillation. $N$ Engl $\mathrm{J}$ Med 2009;361:1139-51.

7 Giugliano RP, Ruff CT, Braunwald E, et al. Edoxaban versus warfarin in patients with atrial fibrillation. N Engl J Med 2013;369:2093-104.

8 Granger $\mathrm{CB}$, Alexander JH, McMurray JJ, et al. Apixaban versus warfarin in patients with atrial fibrillation. $N$ Engl J Med 2011;365:981-92.

9 Patel MR, Mahaffey KW, Garg J, et al. Rivaroxaban versus warfarin in nonvalvular atrial fibrillation. N Engl J Med 2011;365:883-91.

10 Ruff CT, Giugliano RP, Braunwald E, et al. Comparison of the efficacy and safety of new oral anticoagulants with warfarin in patients with atrial fibrillation: a meta-analysis of randomised trials. Lancet 2014;383:955-62.

11 Li XS, Deitelzweig S, Keshishian A, et al. Effectiveness and safety of apixaban versus warfarin in non-valvular atrial fibrillation patients in "real-world" clinical practice. A propensity-matched analysis of 76,940 patients. Thromb Haemost 2017;117:1072-82.

12 Lip GY, Keshishian A, Kamble S, et al. Real-world comparison of major bleeding risk among non-valvular atrial fibrillation patients initiated on apixaban, dabigatran, rivaroxaban, or warfarin. A propensity score matched analysis. Thromb Haemost 2016;116:975-86.

13 Russo-Alvarez G, Martinez KA, Valente M, et al. Thromboembolic and major bleeding events with rivaroxaban versus warfarin use in a real-world setting. Ann Pharmacother 2018;52:19-25.

14 Coleman Cl, Briere J-B, Fauchier L, et al. Meta-Analysis of real-world evidence comparing non-vitamin $\mathrm{K}$ antagonist oral anticoagulants with vitamin $\mathrm{K}$ antagonists for the treatment of patients with non-valvular atrial fibrillation. J Mark Access Health Policy 2019;7:1574541.

15 Kohsaka S, Katada J, Saito K, et al. Safety and effectiveness of apixaban in comparison to warfarin in patients with nonvalvular atrial fibrillation: a propensity-matched analysis from Japanese administrative claims data. Curr Med Res Opin 2018;34:1627-34.

16 Kohsaka S, Murata T, Izumi N, et al. Bleeding risk of apixaban, dabigatran, and low-dose rivaroxaban compared with warfarin in Japanese patients with non-valvular atrial fibrillation: a propensity matched analysis of administrative claims data. Curr Med Res Opin 2017;33:1955-63.

17 Inoue H, Umeyama M, Yamada T, et al. Safety and effectiveness of apixaban in Japanese patients with nonvalvular atrial fibrillation in clinical practice: a regulatory postmarketing surveillance, the STANDARD study. J Arrhythm 2019;35:506-14.

18 Ikeda T, Ogawa S, Kitazono T, et al. Outcomes associated with under-dosing of rivaroxaban for management of non-valvular atrial fibrillation in real-world Japanese clinical settings. J Thromb Thrombolysis 2019;48:653-60.
19 Camm AJ, Fox KAA. Strengths and weaknesses of 'real-world' studies involving non-vitamin $\mathrm{K}$ antagonist oral anticoagulants. Open Heart 2018;5:e000788.

20 Lip GYH. The safety of NOACs in atrial fibrillation patient subgroups: a narrative review. Int J Clin Pract 2019;73:e13285.

21 Pharmaceuticals and Medical Devices Agency. List of Approved products. Available: https://www.pmda.go.jp/english/reviewservices/reviews/approved-information/drugs/0002.html [Accessed Oct 2019].

22 Medical Data Vision Co., Ltd. Introducing MDV database. Available: https://www.mdv.co.jp/solution/pharmaceutical/english/ [Accessed Oct 2019].

23 Austin PC, Stuart EA. Moving towards best practice when using inverse probability of treatment weighting (IPTW) using the propensity score to estimate causal treatment effects in observational studies. Stat Med 2015;34:3661-79.

24 Xu S, Ross C, Raebel MA, et al. Use of stabilized inverse propensity scores as weights to directly estimate relative risk and its confidence intervals. Value Health 2010;13:273-7.

25 Gage BF, Waterman AD, Shannon W, et al. Validation of clinical classification schemes for predicting stroke: results from the National Registry of Atrial Fibrillation. JAMA 2001;285:2864-70.

26 Lip GYH, Nieuwlaat R, Pisters R, et al. Refining clinical risk stratification for predicting stroke and thromboembolism in atrial fibrillation using a novel risk factor-based approach: the Euro Heart survey on atrial fibrillation. Chest 2010;137:263-72.

27 Austin PC. Balance diagnostics for comparing the distribution of baseline covariates between treatment groups in propensity-score matched samples. Stat Med 2009;28:3083-107.

28 Normand ST, Landrum MB, Guadagnoli E, et al. Validating recommendations for coronary angiography following acute myocardial infarction in the elderly: a matched analysis using propensity scores. J Clin Epidemiol 2001;54:387-98.

29 Mamdani M, Sykora K, Li P, et al. Reader's guide to critical appraisal of cohort studies: 2 . assessing potential for confounding. BMJ 2005;330:960-2

30 Ikeda T, Ogawa S, Kitazono T, et al. Real-world outcomes of the Xarelto Post-Authorization Safety \& Effectiveness Study in Japanese Patients with Atrial Fibrillation (XAPASS). J Cardiol 2019;74:60-6.

31 Nielsen PB, Skjøth F, Søgaard M, et al. Effectiveness and safety of reduced dose non-vitamin $\mathrm{K}$ antagonist oral anticoagulants and warfarin in patients with atrial fibrillation: propensity weighted nationwide cohort study. BMJ 2017;356:j510.

32 Inoue H, Umeyama M, Yamada T, et al. Safety and effectiveness of reduced-dose apixaban in Japanese patients with nonvalvular atria fibrillation in clinical practice: a sub-analysis of the standard study. $J$ Cardiol 2020;75:208-15

33 Coleman CI, Peacock WF, Bunz TJ, et al. Effectiveness and safety of apixaban, dabigatran, and rivaroxaban versus warfarin in patients with nonvalvular atrial fibrillation and previous stroke or transient ischemic attack. Stroke 2017;48:2142-9.

34 Amin A, Keshishian A, Trocio J, et al. Risk of stroke/systemic embolism, major bleeding and associated costs in non-valvular atrial fibrillation patients who initiated apixaban, dabigatran or rivaroxaban compared with warfarin in the United States Medicare population. Curr Med Res Opin 2017;33:1595-604.

35 Deitelzweig S, Luo X, Gupta K, et al. Comparison of effectiveness and safety of treatment with apixaban vs. other oral anticoagulants among elderly nonvalvular atrial fibrillation patients. Curr Med Res Opin 2017;33:1745-54.

36 Lin Y-C, Chien S-C, Hsieh Y-C, et al. Effectiveness and safety of standard- and low-dose rivaroxaban in Asians with atrial fibrillation. $J$ Am Coll Cardiol 2018;72:477-85.

37 Cha M-J, Choi E-K, Han K-D, et al. Effectiveness and safety of nonvitamin $\mathrm{K}$ antagonist oral anticoagulants in Asian patients with atrial fibrillation. Stroke 2017;48:3040-8.

38 Huang $\mathrm{H}-\mathrm{Y}$, Lin S-Y, Cheng S-H, et al. Effectiveness and safety of different rivaroxaban dosage regimens in patients with non-valvular atrial fibrillation: a nationwide, population-based cohort study. Sci Rep 2018;8:3451.

39 Lee S-R, Choi E-K, Han K-D, et al. Edoxaban in Asian patients with atrial fibrillation: effectiveness and safety. J Am Coll Cardiol 2018;72:838-53.

40 Ono T, Ikemura N, Kimura T, et al. Contemporary trend of reduceddose non-vitamin $\mathrm{K}$ anticoagulants in Japanese patients with atrial fibrillation: a cross-sectional analysis of a multicenter outpatient registry. J Cardiol 2019;73:14-21.

41 Heine GH, Brandenburg V, Schirmer SH. Oral anticoagulation in chronic kidney disease and atrial fibrillation. Dtsch Arztebl Int 2018;115:287-94. 
42 Malhotra K, Ishfaq MF, Goyal N, et al. Oral anticoagulation in patients with chronic kidney disease: a systematic review and meta-analysis. Neurology 2019;92:e2421-31.

43 Kumar S, de Lusignan S, McGovern A, et al. Ischaemic stroke, haemorrhage, and mortality in older patients with chronic kidney disease newly started on anticoagulation for atrial fibrillation: a population based study from UK primary care. BMJ 2018;360:k342.
44 Jain N, Reilly RF. Clinical pharmacology of oral anticoagulants in patients with kidney disease. Clin J Am Soc Nephrol 2019;14:278-87.

45 Turpie AGG, Purdham D, Ciaccia A. Nonvitamin K antagonist oral anticoagulant use in patients with renal impairment. Ther Adv Cardiovasc Dis 2017;11:243-56.

46 Pisters R, Lane DA, Nieuwlaat R, et al. A novel user-friendly score (HAS-BLED) to assess 1-year risk of major bleeding in patients with atrial fibrillation: the Euro Heart survey. Chest 2010;138:1093-100. 